\title{
GAS BREMSSTRAHLUNG AND ASSOCLATED \\ PHOTON-NEUTRON SHIELDING CALCULATIONS \\ FOR ELECTRON STORAGE RINGS
}

\author{
by \\ J. C. Lu, W. R. Nelson and K. R. Kose \\ Stanford Linear Acceleralor Center \\ Stanford University, Stanford, California 94309
}

\begin{abstract}
The EGS4 electron-photon Monte Carlo code has been used to study the characteristics of the bremsstrahlung $x$-rays generated from the interaction of circulating electrons with the residual gas in accelerator storage rings. Gas bremsstrahlung dose rates are given for various opening angles as a function of the electron beam energy ranging from 0.5-10 GeV. Photon and neutron dose rates, generated from various devices struck by gas bremsstrahlung in a synchrotron radiation beamline, are also presented along with the photon spectral and transmission results. The EGS4-predicted results are found to be in basic agreement with the measurements made at the Stanford Synchrotron Radiation Laboratory. Figures, equations, and a simple method useful for the photos-neutron shielding design for beamlines are provided.
\end{abstract}

* Work supported by the U. S. Deaprtment of Energy under contract DE-AC-0376SF00515. 


\section{INTRODUCTION}

FOR the stored electron beam operation of an accelerator storage ring, gas bremsstrahlung and synchrotron radiation are two types of radiation that have to be considered in the shielding design for the photon beamlines. Figure 1a shows a schematic drawing of a synchrotron radiation beanline originated from a straight section in an electron storage ring. Synchrotron radiation is emitted when the elertrons are bended in a dipole or an insertion device (wiggler or undulator) in the ring while the gas bremsstrahlung photons are produced from the circulating electrons inleracting with the residual gas inside the straight section. Both synchrotron radiation and gas bremsstrahlung will channel into the beamline. Rindi (1982) has given a very good introduction to the theory of gas bremsstrahlung production. The high power (but very low energy) synchrotron photons can generally be shielded easily with lead sheets, but the gas bremsstrahlung is very energetic and is highly collimated in the forward direction. The shielding for gas bremsstrahlung is generally made of tungsten or lead metal blocks (called beamstops) positioned in the zero-degree, median plane of the beamline. The gas bremsstrahlung shielding in forward direction has also been studied previously (Rindi 1982; Ban el al. 1989; Tromba and Rindi 1990, Ferrari et al. 1993). Equally important, but not studied before, are the secondary radiation (scattered photons and photoneutrons) that are generated in and scattered out of a synchrotron radiation beumline when gas bremsstrahlung strikes a device (e-g., slits, mirrors, monochrometers, beamstops, etc). This secondary radiation poses a potential shielding problem particularly for the beamlines of long straight sections in high-energy, high-current storage rings.

In this study the EGS4 electron-photon Monte Carlo code (Nelson et al. 1985) has been used to calculate the gas bremsstrahlung dose rates in the forward angles, using the bremsstrahlung angle sampling algorithm developed by Bielajew et al. (1989). The secondary photon and neutron dose rates from beamline devices struck by gas

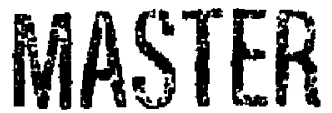

2

DISTRIBUTION OF THIS DOCUMENT IS UNLMMTTED

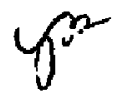


bremsstrahlung were also studied with EGS4. Photon and neutron dose rate measurements around an undulator beamline at the Stanford Synchrotron Radiation Laboratory (SSRL) were made and the results are compared with the EGS4 predictions. Useful figures and equations for the purpose of photon-neutron beamline shielding design are presented.

\section{METHODS}

The geometry used in the EGS4 calculations for studying the gas bremsstrahlung and the secondary photon and neutron radjation is showa in Fig. 1b. The stored electrons, with energy E passing through a cylindrical air path, generate bremsstrahlung photons with a spectrum $d N / d k \propto 1 / k$, where $k$ is the photon energy. The air path is the length of the straight section of the ring in which the device (dipole or insertion device) is located. The gas bremsstrahlung photon fluence crossing a sphere centered at the staning point of the air path is scored as a function of the source angle $\left(\theta_{3}\right)$ relative to the electron beam direction. The bremsstrahlung photons may interact with a device (target) in the beamline, thereby producing secondary phoions and neutrons from the ensuing electromagnetic cascade. The secondary photon fluence crossing a sphere centered at the starting point of the target is scored as a function of the target angle $\left(\theta_{\mathrm{T}}\right)$. In this study the photon fluences scored on the spheres are converted to doses using the maximum and surface $(0-1.2 \mathrm{~cm}$ depth) fluence-to-dose equivalent conversion factors from Rogers (1984). The maximum dose rate is useful for shielding design purpores, whereas the surface dose rate may compare better with the measurements.

Neutrons are not generally produced and transported in the EGS4 code. In this study, howevet, a special subroutine AUSGAB based on photon track-length was created for photoneutron production. The distribution of the differential photon track length inside 
the beamline target, $\mathrm{d} / \mathrm{dk}$ ( $\mathrm{cm} \mathrm{MeV} \mathrm{M}^{-1}$ ), was scored. The neutron yield, $\mathrm{Y}$, from giant resonance reactions, $(\gamma, \mathrm{xn})$, was then obtained using the following equation:

$$
Y=\int d \psi / d k \quad \sigma_{Y, x n}(k) \times d k
$$

where $\sigma_{\gamma, x n}(k)$ is the giant resonance cross section which produces $x$ number of neutrons ( $1 \leq x \leq 3$ in this study). The photoneutron cross sections used in the calculations for different materials were obtained from Dietrich and Berman (1988). In the EGS4 calculations the integration was done by summation over a photon energy range between the reaction thresholds and an upper limit of $40 \mathrm{MeV}$. Neutron fluence at $1 \mathrm{~m}$ away from the target was then oblained from the yield by assuming the target was a point source and the neutron emission was isotropic. These two astumptions produced emors whuse magnitudes depend on the target material and the photon energy. A conversion factor of $3.18 \times 10^{-10} \mathrm{~Sv} \mathrm{~cm}^{2}$ for giant resonant geutrons was used to convert the neutron fluence (either calculated or measured) to the maximum neutron dose.

Neutron production from the quasi-deuteron and photopion reactions which can be induced by photons higher than about $30 \mathrm{MeV}$ was not considered. This should have smal! error because the bremsstrahlung photon spectrum is nearly $\mathbf{k}^{-1}$ and the cross sections for the above two reactions are also smaller than the giant resonance one. Neutron transport was not made either after their productions in our study. This would produce small error because the mean free path of giant resonance neutrons is comparable with the dimensions of the beamline components (except for beamsiops) made of metals with intermediate and high atomic numbers. Since the passible neutron interactions inside the target is not accounted for, the true neutron energy and intensily outside the target could be lower than the calculated ones. 
The electron beam energy $\mathrm{E}$ used in the present work ranged from 0.5 to $10 \mathrm{GeV}$. A pencil beam size was used in the calculations, but the effect of a Gaussian-shaped, finite size beam was also studied. The air target $\left(78.03 \% \mathrm{~N}_{2}, 21.03 \% \mathrm{O}_{2}\right.$, and $0.94 \%$ Ar by

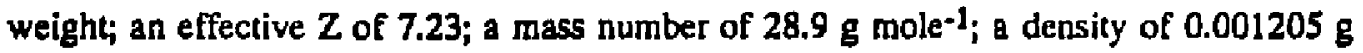
$\mathrm{cm}^{-3}$ ) had a path length of $1 \mathrm{~m}$. A though the residual gas in the vacuum chamber of the ring is generally of ultra-Jow pressure (about $0.1 \mu \mathrm{Pa}$ ), one atmospheric pressure was used in the calculations to increase the statistics in the Monte Carlo. The calculated dose results were then linearly extrapolated to low pressures, a practice verified by Tromba and Rindi (1990). The particle-transport thresholds, i.e., the EGS4 parameters ECUT and PCUT, were set to be $1.511 \mathrm{MeV}$ for charged particles and $0.1 \mathrm{MeV}$ for photons, which were the same as the PEGS4 data-generation parameters AE and AP, respectively. The error in the bremsstrahlung dose from these threshold settings has been shown to be less than 1\% (Ban et al. 1989). The PRESTA algorithm (Bjelajew 1986) was not used in the study, because the transport of low-energy electrons wis not considered to be important.

\section{RESULTS AND DISCUSSIONS}

In this section the EGS4 calculated results and the secondary photon and neutron dose mate measurements made at SSRL are presented and discussed.

\section{Gas bremsstrahlunte}

Several characteristics of the gas bremsstrahlung source term were studied: spectrum, angular distribution, dose rates as a function of stored beam energy, and is attenuation in shield.

An analytical formula from eqn (2.11-3) of Rossi (1952), which can be used to calculate the gas bremsstrahlung spectrum, is given by: 


$$
k d N / d k=4 \alpha r_{c}^{2}\left(2 N_{A} / A\right) Z(Z+1) f(v, Z),
$$

and $\quad f(u, Z)=\left(v^{2}-4 v / 3+4 / 3\right) \ln \left(183 Z^{-1 / 3}\right)+(1-v) / 9$

where

$\alpha$ is fine structure constant $=1 / 137$;

$I_{E}$ is classical electron radius $=282 \times 10^{-13} \mathrm{~cm}$;

$\mathrm{N}_{\mathrm{A}}$ is the Avogadro constant $=6.02 \times 10^{23}$ and the multiplier 2 accounts for the fact that over $99 \%$ of air is made of diatomic molecules;

For air, $\mathrm{A}=28.9 \mathrm{~g} \mathrm{~mole}^{-1} ; \mathrm{Z}=7.23$;

$v=\mathbf{k J}$.

$\mathrm{dN} / \mathrm{dk}$ is the number of photons within $\mathrm{dk}$ about $\mathrm{k}$ when one electron with energy $\mathrm{E}$ passes through an air path of $1 \mathrm{~g} \mathrm{~cm}^{-2}$ thick. An EGS4-calculated spectrum resulting from one electron of $1 \mathrm{GeV}$ passing through an air path of $0.1205 \mathrm{~g} \mathrm{~cm}^{-2}$ is shown in Fig. 2, where it is compared on an absolute basis with that predicted from egn (2). The agreement is very good, except the equation fails to account for the fact that the cross section drops to zero when $k$ approaches $E$. The bremsstrahlung spectrum, dN/dk, is also observed to be close to $\mathbf{k}^{-1}$ for photons with energy less than $4-5 \%$ of beam energy $E$; an observation agrees with that of Ban et al. (1989).

The number of photons generated per electron is found to be in good agreement (within 4\%) with that calculated from eqn (1) of Tromba and Rindi (1990), which was atso based on EGS4.

In the defautt version of EGS4 the photon energy is properly samp'ed but the production angle is fixed at $\theta_{B}=m / E=0.511 / E$, the so called "characteristic angle". This is acceplable for most shower studies because, in general, multiple Coulomb scattering angles dominate over the bremssimhlung production angles. However, for very thin targets, such as the gas bremsstrahlung case, the reverse may be true. Therefore, the latest 
bremsstrahlung angle sampling algorithm de Jeloped by Bielajew et al. (1989) for EGS4 was used in this study. In Fig. 3 the effect of properly sampling the production angle is demonstrated in terms of the photon dose rate as a function of the source angle. The default EGS4 gives an artificial peak at $\theta_{5}=0.511 / 500=0.06^{\circ}$, whute the correct sampling peaks at $0^{\circ}$ and falls rapidly at wider angles, as expected.

Figure 3 also shows the dependence of the dose rate on the source angle. A detector subiending an opening angle, $\theta_{d}$ will measure an average dose rate over the opening angle. To show the importance of the detector size, the maximum dose rate is plotted as a function of beam energy for two opening angles in Fig. 4. At a downbeam distance of 10 meters from the gas bremsstrahlung air source, the one with the larger opening angle $\left(\theta_{d}=\right.$ $\left.0.15^{\circ}\right)$ represents a detector with a $2.54 \mathrm{~cm}$ radius and the smaller one $\left(\theta_{d}=0.01^{\circ}=\right.$ the smallest angle bin in this study) represents a detector with a $0.18 \mathrm{~cm}$ radius. Note that, at $1 \mathrm{GeV}$ beam energy, the dose rates measured by these two detectors differ by about three orders of magnitude. Also shown are dose rate formulae proposed by Tromba and Rindi (1990) and Ferrari et. al. (1993). Tromba and Rindi used the default version of EGS4 and their results are comparable to those of our smaller detector. However, the results of Ferrari were about a factor of two higher than ours. The reason for the difference is explained below.

There are two factors that will affect the dose rate distributions (shown in Figs. 3 and 4) in an opposite way. First, the multiple scattering in the ulura-low-pressure residual gas should be negligible**. However, since $1-\mathrm{atm}$ and $1 \mathrm{~m}$ of air path (equals to $3.3 \times 10^{-3} \mathrm{X}_{\mathrm{o}}$ ) was used in the EGS4 calculation, the dose rate in the very-forward angles without multiple scattering will be higher than those in Figs. 3 and 4. Ferrari et al. (1993) showed that it is about a factor of two for an example he used (this reflects the difference in Fig. 4). Secondly, the fact that the electron beam has finite size and may tend to be Gaussian in shape will flatten out the dose peak and, therefore, lower the dose rate. This has been demonstrated in one of our study using the $3 \mathrm{GeV}$ and the Gaussian-shaped SSRL betm. 
It is because of these intricate reasons (the detector opening angle, the beam size and shape, and the multiple scattering) that the bremsstrahlung dose rate in the very-forward angles derived in this manner have to be used wisely. A beller, but complicated, way to design the shielding needed for the gas bremsstrablung in the forward angle is to model the shielding (e.g., beamstop) together with the air path in the beamline and calculate the average dose over an area of interest behind the shielding. In this case, multiple scattering inside the shielding will dominate other factors and, thus, the angular cistribution after the shielding.

For gas bremsstrahlung shielding, an attenuation length ranging from 19 to $25 \mathrm{~g} \mathrm{~cm}^{-2}$ were obtained before (Tromba and Rindi 1990; Schopper 1990). In this study the dose rate (average over a forward angle of $0.01^{\circ}$ ) after the target (see Fig. 1) was determined as a function of target thickness. From the plot of the calculated dose rate vs, the target thickness, an attenuation length of $21 \mathrm{~g} \mathrm{~cm}^{-2}$ for lead was derived. Therefore, a $\lambda$ value of $24 \mathrm{~g} \mathrm{~cm}^{-2}$ for lead (corresponding to the Compton minimum of the attenuation coefficient) is conservative and appropriate for shielding design purposes.

\section{Example of Use of the Results}

The gas bremsstrahlung dose rates in Fig. 4 have been nomalized to a beam current of $1 \mathrm{~A}$ and an air path of $1 \mathrm{~m}$ with a vacuum pressure of $1 \mu \mathrm{Pa}$ (7.52 ntorr). For an undulator line at SSRL ( $3 \mathrm{GeV}, 0.1 \mathrm{~A}$, and a $4.75 \mathrm{~m}$ long straight section wath a pressure of $0.5 \mu \mathrm{Pa}$ ), the dose rate in the beamline is estimated to be $0.9 \mathrm{~Sv} \mathrm{~h}^{-1}$ over an angle of $0.01^{\circ}$, and $0.2 \mathrm{mSv} \mathrm{h}^{-1}$ over an angle of $0.15^{\circ}$. Because storage rings are running continuously, a shielding design limit of $5 \mu \mathrm{Sv} \mathrm{h}^{-1}$ over $2000 \mathrm{~h}$ per year from the gas bremsstrahlung radiation is generally used for occupational workers (some facilities may use a more conservative value of $0.5 \mu \mathrm{Sv} \mathrm{h} \mathrm{h}^{-1}$ ). Using $0.9 \mathrm{~Sv} \mathrm{~h}^{-1}$ at small angles as the limit, this implies a shielding with an attenuation factor of $5 \times 10^{-6}$ is needed. Using the above attenuation factor and attenuation length $\lambda$, a maximum lead shielding of $25 \mathrm{~cm}$ thick in the forward direction is required for the SSRL undulator line. 


\section{Secondary Photons}

Generally, gas bremsstrahlung is inherently well shielded in the forward direclion by mirrors, monochrometers, beamstops, and other devices in the beamline. Gas bremsstrahlung will produce electromagnetic showers in these devices, resulting in secondary radiation (scatlered photons and photoneutrons).

The dose rate due to photon leakage from three sizes of cylindrical lead targets is plotted as a function of the target angle, $\theta_{\mathrm{T}}$, in Fig. 5 . It shows that the dose rate in the backward direction $\left(\theta_{\mathrm{T}} \geq 90^{\circ}\right)$, particularly for $\theta_{\mathrm{T}} \geq 145^{\circ}$, is less insensitive to target size. The effect of electron beam energy on the angular dose distribution is shown in Fig. 6 for a lead target (about $20 \mathrm{~cm}$ in both diameter and length); this trend is also observed in other materials. Note that the photon dose rates in Figs. 5 and 6 are at the surface and corresponding to a gas bremsstrablung source term produced in $1 \mathrm{~m}$ of air at $1 \mathrm{~atm}$. As seen from Figs. 5 and 6 , the photon dose rates at $\theta_{T} \geq 145^{\circ}$ (indicated by the horizontal lines in Fig. 6) can be used to estimate the dose rate in the backward direction within a factor of 2-3. Therefore, curves for estimating the backscattered $\left(\theta_{T} \geq 90^{\circ}\right)$ dose rates from large blocks of different target materials struck by gas bremsstrahlung from electron beams of various energies are shown in Fig. 7. Formulae from fitting the curves for estimating the photon dose rate are also shown below:

$$
\begin{aligned}
& H_{s}=0.35 E^{-0.33} \text { for tungsten } \\
& H_{s}=0.32 E^{-0.36} \text { for lead } \\
& H_{s}=0.23 E^{-0.49} \text { for copper } \\
& H_{s}=0.11 \mathrm{E}^{-0.69} \text { for silicon and aluminum }
\end{aligned}
$$

where $H_{3}$ is the photon surface dose rate $\left(\mu \mathrm{Sv} \mathrm{h} \mathrm{h}^{-1} \mathrm{~A}^{-1} \mathrm{GeV}^{-1} \mu \mathrm{Pa}^{-1} \mathrm{~m}^{-1}\right)$ at 1 in lateral from the beamline target, normalized to a beam current of $1 \mathrm{~A}$, per GeV beam energy, and an air path of 1 meter with a vacuum pressure of $1 \mu \mathrm{Pa}$. E is the electron beam energy in 
GeV. The maximum photon dose rates are about a factor of 2 higher than the surface ones at these target angles. For the SSRL undulator line, the calculated photon dose rate at $1 \mathrm{~m}$ from a large lead beamstop is $0.15 \mu \mathrm{Sv} \mathrm{h} \mathrm{h}^{-1}$, which is about two times higher than the natural photon background.

The reason that the normalized dose rate lines in rig. 7 are not horizontal is because that the higher the beam energy, the deeper the location of the shower maximum and, therefore, photons will be altenusted more as they travel backwards out of the target. The secondary photon (and neutron also) dose rates are expected, and have been demonstrated in this study, not to be dependent on the target's dowabeam distance from the air source (see Fig. 1).

Knowledge of the energy distribution of the scattered photons is important for lateral shielding design. Shown in Fig. 8 are EGS4-calculated spectra emanating into two angular bins for 3-GeV gas bremsstrahlung striking a large lead target. As expected, the spectrum at $90^{\circ}-95^{\circ}$ is slightly harder than that at $130^{\circ}-140^{\circ}$. Both spectra are within about $10 \mathrm{MeV}$ and have noticeable $0.511 \mathrm{MeV}$ peaks from positron annihilation inside the target. The transmission of the two photon spectra through lead and iron shields is given in Fig. 9. This transmission was calculated using a point-kemel integration technique (Nelson and LaRiviere 1984). For small shield tnicknesses the lower energy photons are absorbed preferentially. For large shield thicknesses the $\lambda$ 's again approach the Compton minimum of the shield (24 $\mathrm{g} \mathrm{cm}^{-2}$ for lead and $34 \mathrm{~g} \mathrm{~cm}^{-2}$ for iron), which demonstrates the dominance and importance of few-MeV photons in the shielding transmission.

\section{Neutrons}

The normalized neutron dose rates at $1 \mathrm{~m}$ away from the target and the neutron yield within the target are plotted in Fig. 10 as a function of the target atomic number. These curves refer to targets with sufficient sizes ( $30 \mathrm{X}_{\mathrm{o}}$ long and $30 \mathrm{X}_{\mathrm{m}}$ in diameter; $\mathrm{X}_{\mathrm{m}}$ is the Moliere unit") to gencrate maximum neutron yields. A least-square fit to the five-point 
line in Fig. 10 would give a neutron yield proportional to $\mathrm{Z}^{0.8}$. However, it is to be noted that Fig. 10 gives only rough estimation when it is used to extrapolate to materials with other atomic numbers. For example, the neutron yield from silicon (a common material for monochrometers and mitrors) is only about half of that from aluminum. For the SSRL undulator line mentioned above, the geutron dose rate at $1 \mathrm{~m}$ from a large lead beamstop is $0.4 \mu \mathrm{Sv} \mathrm{h}^{-1}$, which is about 50 times higher than the natural neutron background.

For smaller targets one can use curves similar to those in Fig. 11 calculated in this study for lead to determine the relative yield as a function of target length. For example, the relative yield is 0.9 for a lead target ( $30 \mathrm{X}_{\mathrm{ma}}$ in diameter) with a length of $10 \mathrm{X}_{0}, 11.7 \mathrm{X}_{0}$, 12.7X , and $14 \mathrm{X}_{0}$, for an electron energy of $0.5 \mathrm{GeV}, 1 \mathrm{GeV}, 3 \mathrm{GeV}$, and $10 \mathrm{GeV}$, respectively. The relative yield as a function of target diameter is more complicated, due to the fact that the bremsstrablung photons already have an angular spread and, thus, a finite size, when they hit the devices. The magnitude of the bremsstrahlung beam size depends on the elactron beam size, beam energy, the air thickness, and the downbeam distance of the device. For a first-order approximation, the relative neutron yield is $\geq 0.9$ for a long target with a djameter $\geq 8 \mathrm{X}_{\mathrm{m}}$. For the estimation of neutron yield (and photon dose also) from small targets (either in length or diameter), Monte Carlo calculation is still the most satisfactory approach.

\section{Simple Scaling Method}

A simple scaling method of estimating the neutron jields from large targets struck by gas bremsstrahlung without using EGS4 calculation was developed from this study. It is based on the following two principles. Firsh for thin-target bremsstrahlung, the fractional energy transferred from an electron to photons, $\mathrm{dE} / \mathrm{E}$, is equal to the ratio of the thickness, $t$, to the radiation length of the target, i.e., $d E / E=t / X_{0}$. This relationship can also be derived by integrating eqn (2). This principle obviously is applicable to the case of gas bremsstrahlung. Therefore, the fractional energy (or power) transferred from the cicculating electrons to gas bremsstrahlung pholons is $=t / X_{0}$, where $t$ is the air path length 
and $X_{0}$ for air is $=36.818 \mathrm{~g} \mathrm{~cm}^{-2}$. The second principle is that the neutron yield from a device is not dependent on whether it is electron or gas bremsstrahlung photon that is hitting the device, but is dependent on the radiation power only". Since the data of the maximurn neutron yields from electrons hitting various thick targets are avaibble in Swanson (1979), a simple scaling method based on the two principles can be used to oblain the neulron yields from thick targets hit by gas bremsstrahlung. For example, the power of the circulating electrons $(0.1 \mathrm{~A}$ at $3 \mathrm{GeV})$ at SSRL is $300 \mathrm{MW}$. The air path (4.75 $\mathrm{m}$ with $0.5 \mu \mathrm{Pa}$ ) of the undulator line at SSRL is $2.8 \times 10^{-12} \mathrm{~g} \mathrm{~cm}^{-2}$. The ratio of the air path to air's radiation length is then $7.6 \times 10^{-14}$. Using the first principle, it means that the power transferied to bremsstrahlung photons is $300 \mathrm{MW} \times 7.6 \times 10^{-14}=2.28 \times 10^{-8} \mathrm{~kW}$. A maximum neutron yield of $1.7 \times 10^{12} \mathrm{~s}^{-1}$ for tungsten hit $\mathrm{b} / 1 \mathrm{~kW}$ of electrons is available from Swanson (1979). Using the second principle, this yield is also applicable to $1 \mathrm{~kW}$ of gas bremsstrahlung photons hitting the same device. Therefore, the neutron yield from a tungsten beamstop hit by the photon power is $2.28 \times 10^{-8} \mathrm{~kW} \times 1.7 \times 10^{12} \mathrm{~s}^{-1} \mathrm{~kW}^{-1}=$ $3.9 \times 10^{4} \mathrm{~s}^{-1}$, resulting in a neutron fluence of $0.31 \mathrm{~cm}^{-2} \mathrm{~s}^{-1}$ at $1 \mathrm{~m}$ away from the beanstop. Using the fluence-to-dose conversion factor for giant resonant neutrons, this fluence rate corresponds to a neutron dose rate of $0.35 \mu \mathrm{Sv} \mathrm{h}^{-1}$. This value compares favorably with the EGS4-calculated and the measured values, as shown in the following section.

\section{Measurements and comparison}

An experiment was performed to measure the photon and neutron dose rates from two devices struck by gas bremsstrahlung in a SSRL undulator beamline. The devices were copper slits and a tungsten beamstop. Since the photon and neutron dose rates from the targets were very low, a sensitive $\mathrm{GM}$ counter and a moderated $\mathrm{BF}_{3}$ detector were used for the measurements. They were positioned latersl (about $90^{\circ}$ ) to the target. For the cas $s$ of slits, the measured dose jate was the difference of the dose rates between the closed slits and the open slits. Because the true photon spectrum was not well-known, a 
calibration factor of 0.16 counts $\mathrm{cm}^{2}$ for the ${ }^{226} \mathrm{Ra}$ photons was used for the GM counter and a conversion factor of $3.5 \times 10^{-12} \mathrm{~Sv}^{2}$ for $1.25 \mathrm{MeV}$ photons (Rogers 1984) was used to relate the measured photon fluence to the surface dose. The total error associated with the energy response of the GM counter and the use of the above factors is about $50 \%$. A calibration factor of $\mathbf{1 1 . 2}$ counts $\mathrm{cm}^{2}$ for giant resonant neutrons from the radioisotope neutron source calibrations (Liu et al. 1991) was used for the moderated $\mathrm{BF}_{3}$ deteclor. The error from the neutron energy response of the $\mathrm{BF}_{3}$ detector is about $20 \%$.

To simplify the EGS4 modeling, the geometries of the devices were simulated with cylindrical targets in different sizes; $12.1 \times 3.0$ for copper slits and 10.2x3.0 for tungsten beamstop (numbers are length times radius in $\mathrm{cm}$ ). According to the calculated relative neutron yield curves, the size of the tungsten beamstop ( $29 \mathrm{X}_{\mathrm{o}}$ and $4 \mathrm{X}_{\mathrm{m}}$ ) would produce the maximum neutron yield while the copper slits $\left(8.4 X_{0}\right.$ and $\left.2.4 X_{m}\right)$ would have about $70 \%$ of its maximum yield. The components and beampipe around the device were not modeled in the calculations. Neutron transport was not made either. The photon dose rale is calculated to be the mean dose rate averaged over the target angles subtended by the GM counter. The fact that the atomic number of the residual gas was not well known may contribute about $30 \%$ error to the calculated results. The total errors for the calculated results were estimated to be around $50 \%$ for photons and higher for neutrons.

In Table 1 the measured dose rate results, in comparison with the EGS4 calculations, are shown. The calculated results for photon using eqn (3) and neutron using the simple scaling method are also shown. All results are referred to the following gas bremsstrahlung source parameters during the measurements: $3 \mathrm{GeV}$ electron beam, $0.1 \mathrm{~A}$ current, and an average gas pressure of $0.5 \mu \mathrm{Pa}$ in the $4.75 \mathrm{~m}$ long straight section of the undulator. Of all parameters the vacuum pressure was the most difficult one to be measured and had the largest uncertainty (probably a factor of two). Therefore, the errors for the measured results were about a factor two. Considering the complication in the comparison, the agreement among three methods is good (within a factor of two). 
In Table 1 the photon dose rales are about 2 times higher than the na:ural photon background level while the neutron dose rates are about $20-50$ times higher than the neutron background. The total (phuton and neutron) dose rates are about 4 times higher than the natural background (about $1 \mathrm{mSv} \mathrm{y}^{-1}$ ).

\section{CONCLUSIONS}

An EGS4 user code has been developed to study the gas bremsstrahlung and its interaction with the beamline devices. The characteristics of the gas bremisstrahlung; the spectrum and the dose rate as a function of angle and electron beam energy, are piesented. The photon and neutron dose rates (and the photon spectra) generated from various devices hit by gas bremsstrahlung are also summarized. Useful figures and equations for beamline shielding d:sigo are given. A simple scaling method is also proposed to be used to estimate the neutron dose. The fact that the EGS4-calculated results are in basic agreement with the measurements indicates that the presented figures, equations and simple method should provide similar facilities a first cut model for estimating the photon and neutron dose mates in a synchrotron radiation beamline in most situations.

Both measured and calculated results for an undulator line at SSRL show that the total dose rate around a beamline target could be a few tenths of $1 \mu S v h^{-1}$. If it is assumed that the facility nuns continuously for the whole year and the beamline users occupy the area one-fourth of the time, the personnel exposure will be about the same as the annual natural background and close to the limit of the non-occupational worker. As the regulatory dose limis become lowei and lower and/or the capacity (energy, current, and long straight sections) of storge rings becomes higher and higher, the important shielding design for synchrotron radiation beamlines will become a challenging task in the future. More accurate estimations of the secondary photon and neutron dose rates may 
also be necessary to meet the requirements. More refined work is currently underway by developing a coupled neutron-electromagnetic code for further studies.

Acknowledgments - This work was supported by the U.S. Department of Energy under contract DE-AC-03-76SFo0S15. The assistance from $R$. Seefred and SSRL staff, $M$. Rowen and D. Neal, during the measurements are greally appreciated. The discussions on the EGS4 simulations with S. Mro and N. Ipe of SLAC and on the vacuum pressure with B. Scott and H. Morales of SSRL are also acknowledged.

\section{REFERENCES}

Ban, S.; Hirayama, H.; Miura, S. Estimation of absorbed dose due to gas bremsstrahlung from electron storage rings. Health Phys. 57:407-412; 1989.

Bielajew. A. F.; Mohan, R.; Chui, C-S. Improved bremsstrahlung photon angular sampling in the EGS4 code system. National Research Council of Canada; NRCC Internal Report, PIRS-0203; 1989.

Bielajew. A. F.; Rogers, D. W. O. PRESTA - the Parameter Reduced Electron Step Transport Algorithm - for electron Monte Carlo transport. Ottawa, Canada; National Research Council of Canada; NRCC Internal Repor, PIRS-042; 1986.

Dietrich, S. S.; Berman, 1. B. Atlas of photoneutron cross sections obtained with monoenergetic photons. Atomic Data and Nuclear Data Tables. 38:199-338; 1988.

Ferrari, A.; Pelliccioni, M.; Sala, P.R. Estimation of fluence rate and absorbed dose rate due to gas bremsstrahlung from electron storage rings. Nucl. Instr. Meth. B83:518-524; 1993.

Liu, J. C.; Jenkins, T. M.; McCall, R. C. and Ipe, N, E. Neutron dosimetry at SLAC: neutron sources and instrumencation. Stanford, $\mathrm{CA}_{;}$Stanford Linear Accelerator Center, SLAC-TN-91-3; 1991. 
Nelson, W. R.; Hirayama, H.; Rogers, D. W. O. The EGS4 code system. Stanford, CA; Stanford Linear Accelerator Center; SLAC-265; 1985.

Nelson, W. R.; LaRiviere, P. D. Primary and leakage radiation calculations at 6, 10, and 25 MeV. Health Phys. 47:811-818; 1984.

Rindi, A. Gas bremsstrahlung from electron storage rings. Health Phys. 42:187-193; 1982.

Rogers, D. W. O. Fluence to dose equivalent conversion factors calculated with EGS3 for electrons from $100 \mathrm{keV}$ to $20 \mathrm{GeV}$ and photons from $11 \mathrm{keV}$ to $20 \mathrm{GeV}$. Health Phys. $46 ; 891-914 ; 1984$.

Rossi, B. High-energy paricles. Prentice-Hall, Jnc.; Englewood, N.J. ; 1952

Schopper, H. Numerical data and functional relationships in science and technology. New Series, Group I: Nuclear and particle physics, Vol. 11, Shielding against high energy radiation. Landoli-Bomstein, Geaeva; 310-313; 1990.

Swanson, W. P. Radiological safety aspects of the operation of electron linear accelerators. Vienna; Intemational Atomic Energy Agency, IAEA; IAEA Technical Report Series No. 188; 1979.

Tromba, G.; Rindi, A Gas bremsstrahlung from electron storage rings: a Monte Carlo evaluation and some useful formulae. Nucl. Instr. Meth. A292:700-7U5; 1990. 
Fig.1 a) a schematic drawing of a synchrotron radiation beamline which contains both the synchrouron radiation and the gas bremsstrahlung photons originating from a straight section inside the electron storage ring. Beamline devices, e.g., slits, mirror, and beamstop, are also shown.

b) EGS4-geometry for studying the gas bremsstrahlung resulting from electrons with an energy $E$ interacting with an air source (the straight section) and the secondary photon and neutron radiation from a beamline device struck by gas bremsstrahlung. Note the two spheres and the two types of angles, $\theta_{5}$ and $\theta_{T}$, are used for photon fluence scoring.

Fig. 2 Absolute comparison between an EGS4-generated bremsstrahlung spectrum and that given by eqn (2), resulting from one $1-\mathrm{GeV}$ electron interacting with 0.1205 $\mathrm{g} \mathrm{cm}^{-2}$ of air.

Fig. 3 The effect of bremsstrahlung angle $\left(\theta_{B}\right)$ sampling on the dose rate as a function of the source angle $\left(\theta_{S}\right)$ for an electron energy of $0.5 \mathrm{GeV}$. An artifact is observed at $\theta_{S}=0.511 / 500=0.06^{0}$ when tly: default EGS4 method is used.

Fig. 4 Bremsstrahlung dose rate as a function of electron beam energy for detectors of two different sizes. Comparisons are also made with formulae by Tromba and Rindi (1990) and Ferrari et al. (1993) which covered 0.1-1 GeV. See text for the explanation of the effects on the dose distributions of Figs. 3 and 4 from both the multiple scattering in the residual gas and the finite-size, Gaussian-shaped electron beam.

Fig. 5 The secondary photon dose rate from three cylindrical lead targets of different sizes (dimensions are length times diameter) struck by 3-GeV gas bremsstrahlung as a function of the fead-target angle $\left(\theta_{\mathrm{T}}\right)$. The dose rates at $\theta_{\mathrm{T}} \geq 145^{\circ}$ are observed to be insensitive to enget size. 
Fig. 6 The secondary photon dose rate from a cylindrical lead target (length and diameter are both $20 \mathrm{~cm}$ ) as a function of target angle $\left(\theta_{\mathrm{T}}\right)$ for electron beam energies ranging from 0.5 to $10 \mathrm{GeV}$. The horizontal lines indicate the dose rates that can be used to estimate the dose in the backward direction $\left(\theta_{T} \geq 90^{\circ}\right)$ within a factor of 2-3.

Fig. 7 The secondary photon dose rate at $1 \mathrm{~m}$ lateral from large targets of five materials as a function of the electron beam energy. Equations from fitting the lines for estimating the backscattered dose rates are shown as eqns (3a-3d) in text. The values are normalized to a beam current of $1 \mathrm{~A}$, per GeV beam energy, and an ait path of $1 \mathrm{~m}$ with a pressure of $1 \mu \mathrm{Pa}$.

Fig. 8 The secondary photon spectra emitted into two target angle bins from a lead target struck by gas bremsstrahlung. The spectra and their transmission in shields are dominated by photons with energies of a few $\mathrm{MeV}$.

Fig. 9 The transmission and the attenuation length $(\lambda)$ of secondary photors in lead and iron shields, which show the importance of the photons with energies around the Complon-minimum of the shield.

Fig. 10 The neutron dose rate at 1 meter away from a beamline device struck by gas bremsstrahlung and the neutron yield within the device as a function of the atomic number of the device. The values are normalized to a beam current of $1 \mathrm{~A}$, per $\mathrm{GeV}$ beam energy, and an air path of $1 \mathrm{~m}$ with a pressure of $1 \mu \mathrm{Pa}$.

Fig. 11 The relative neutron yield as a function of the target length in units of radiation length for a cylindrical lead target $\left(30 \mathrm{X}_{\mathrm{m}}\right.$ in diameter) struck by gas bremsstrahlung from four electron beam energies.

\section{DISCLAIMER}

This report was prepared as an account of work sponsared by an agency of the United States Government. Neither the United States Government nor any agency thereof, nor any of their employees, makes any warranty, express or implied, or assumes any legal liability or responsibility for the accuracy, completeness, or usefulness of any information, apparatus, product, or process disclosed, or represents that its use would not infringe privately owned rights. Reference herein to any specific commercial product, process, or service by trade name, trademark, manufacturet, or otherwise does not necessarily constitute or imply its endorsement, recommendation, or favoring by the United States Government or any agency thereol. The views and opinions of authors expressed herein do not necessarily state or reflect those of the United States Government or any agency thereof. 
Table 1 Co.nparison between the EGS4-calculations and the measurements of the photin and neutron dose rates $\left(\mu \mathrm{Sv} \mathrm{h}^{-1}\right)$ at $1 \mathrm{~m}$ lateral from two devices struck by gas bremsstrahlung in a SSRL undulator beamline (source parameters are $3 \mathrm{GeV}$ electron beam, $0.1 \mathrm{~A}$ cursent, and an air pressure of $0.5 \mu \mathrm{Pa}$ in the $4.75 \mathrm{~m}$ long straight section). Estimated dose rates for pholon using eqn (3) and for neutron using the scaling method (see text) are also given.

\section{Footnotes}

** The thickness at which the angles from the multiple scatiering and the bremsstrahlung are equal is about $1.4 \times 10^{-3} \mathrm{X}_{\mathrm{o}}$

- Moliere unit, $X_{m}$ and the radiation length, $X_{0}$, for a material are two quantities used to describe the lateral and longitudinal distributions, respectively, of the propagation of the shower cascade inside the material.

\# This implies that the photon track length distribution between the photo-neutron thresholds and $40 \mathrm{MeV}$ within the target is the same for a target hit by electrons or bremsstrahlung photons. 
(a)

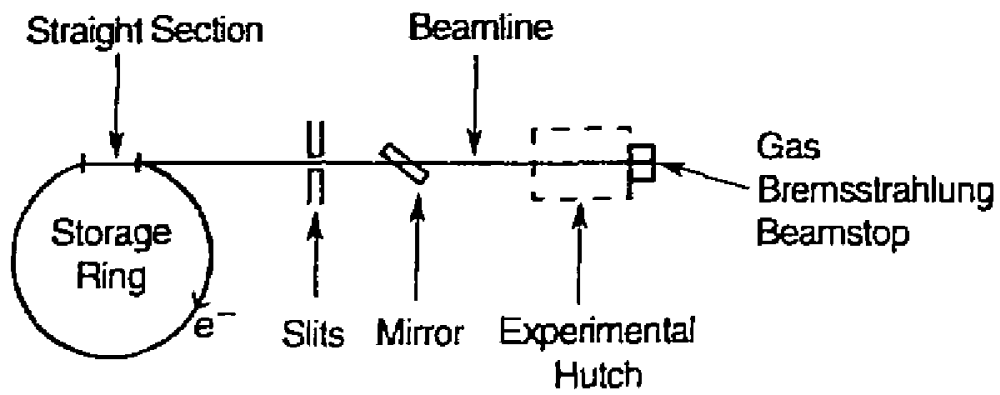

(b)

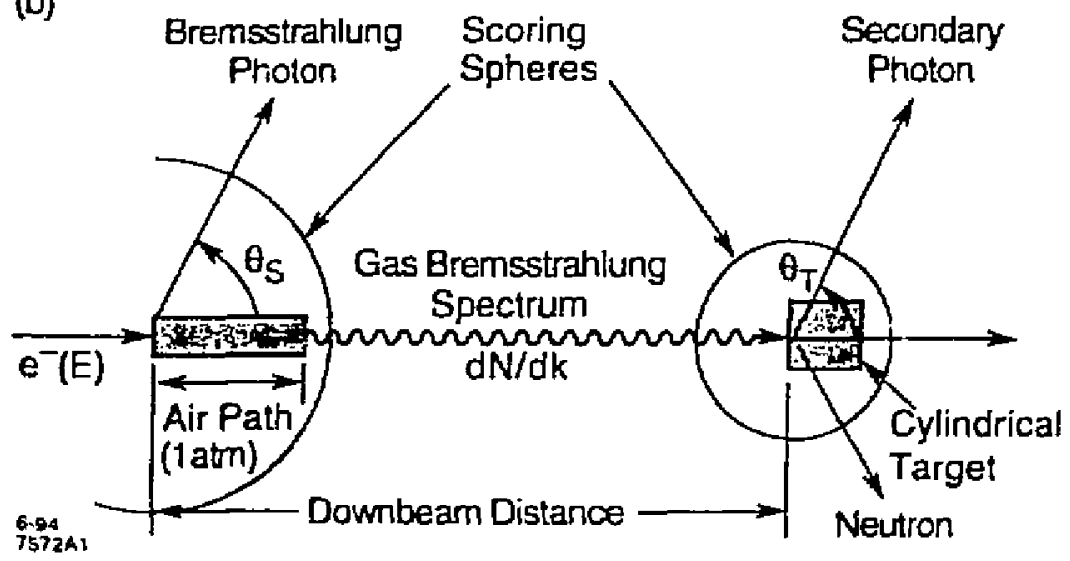

Fig. 1 


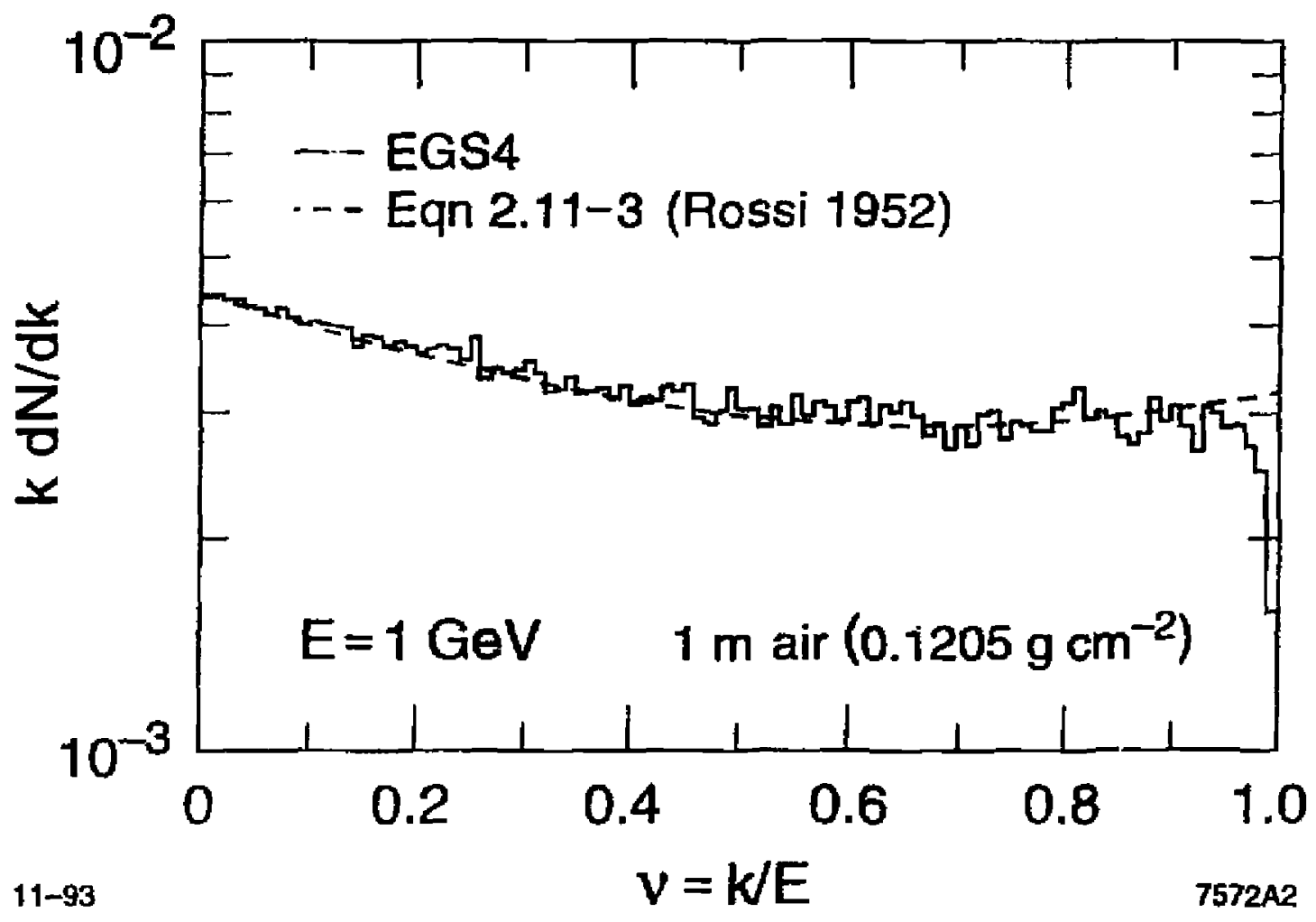

Fig. 2 


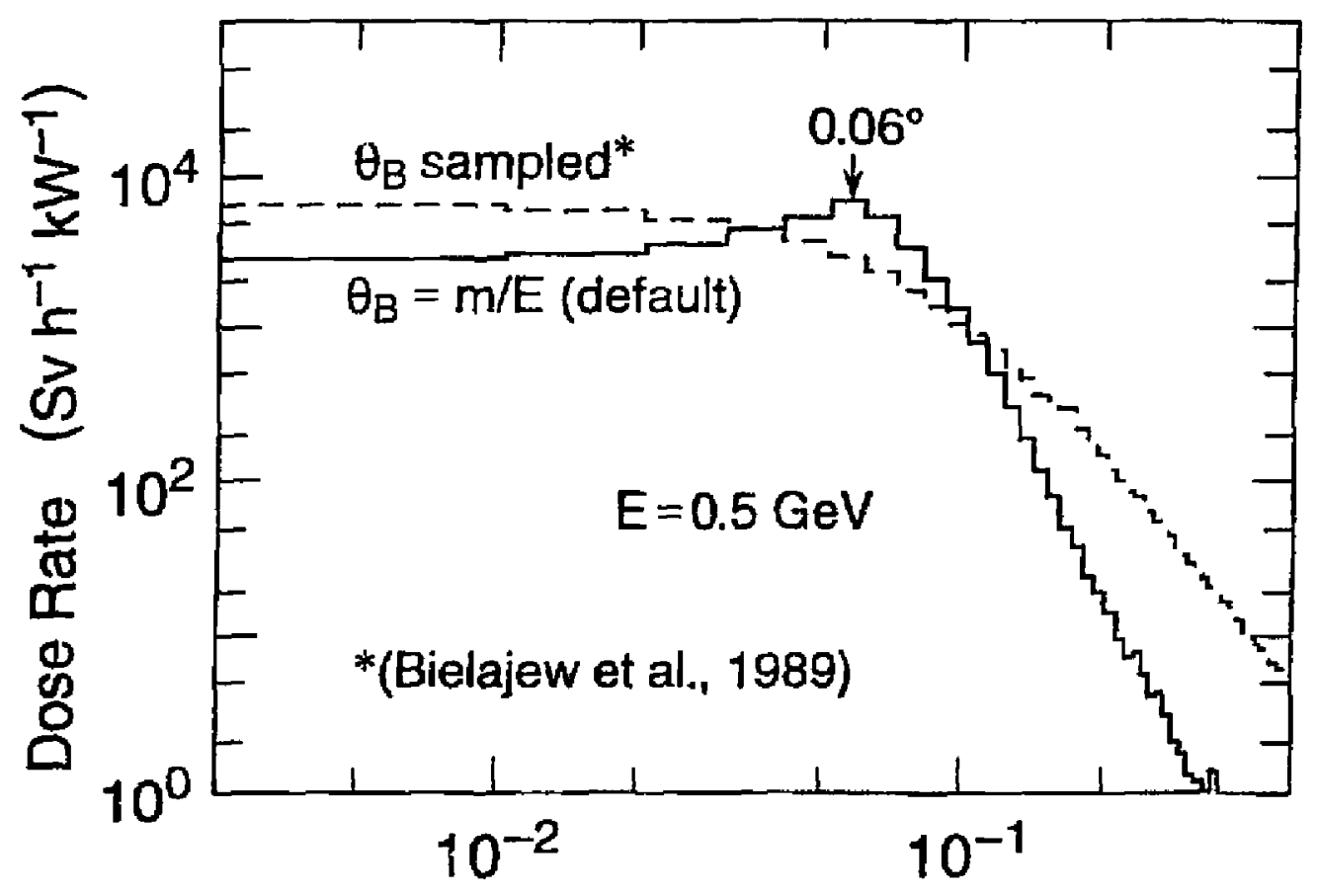

Fig. 3 


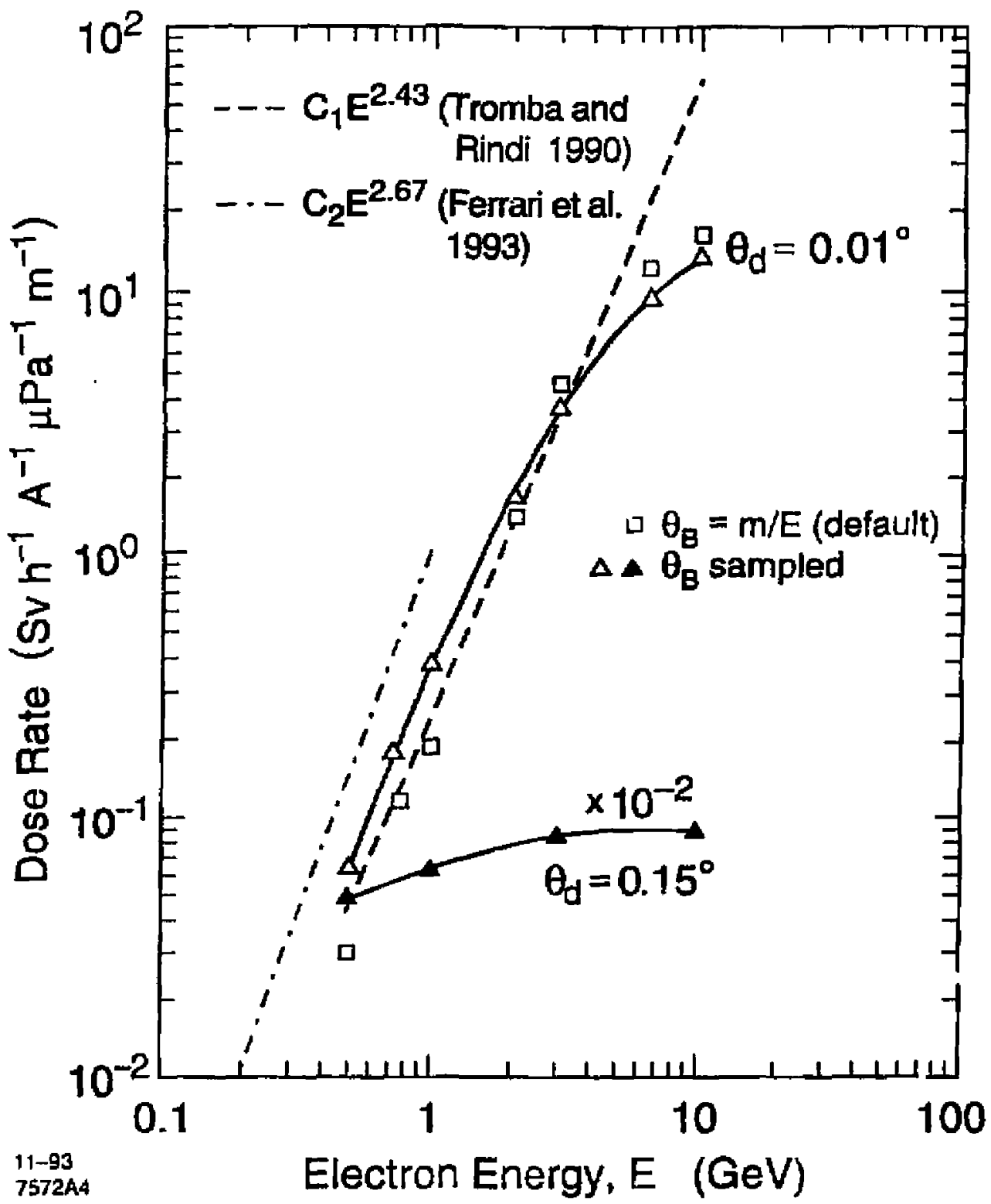

Fig. 4 


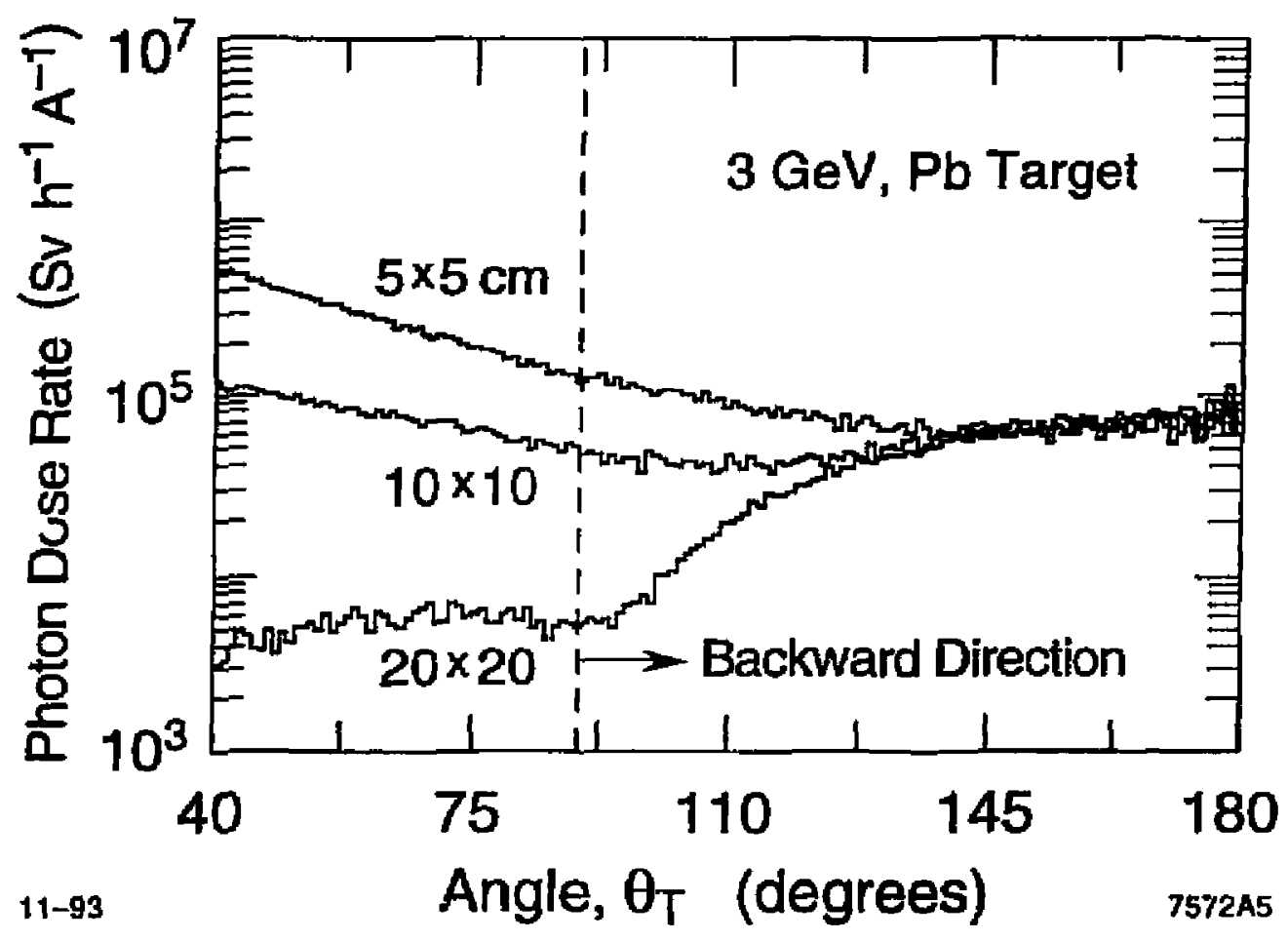

Fig. 5 


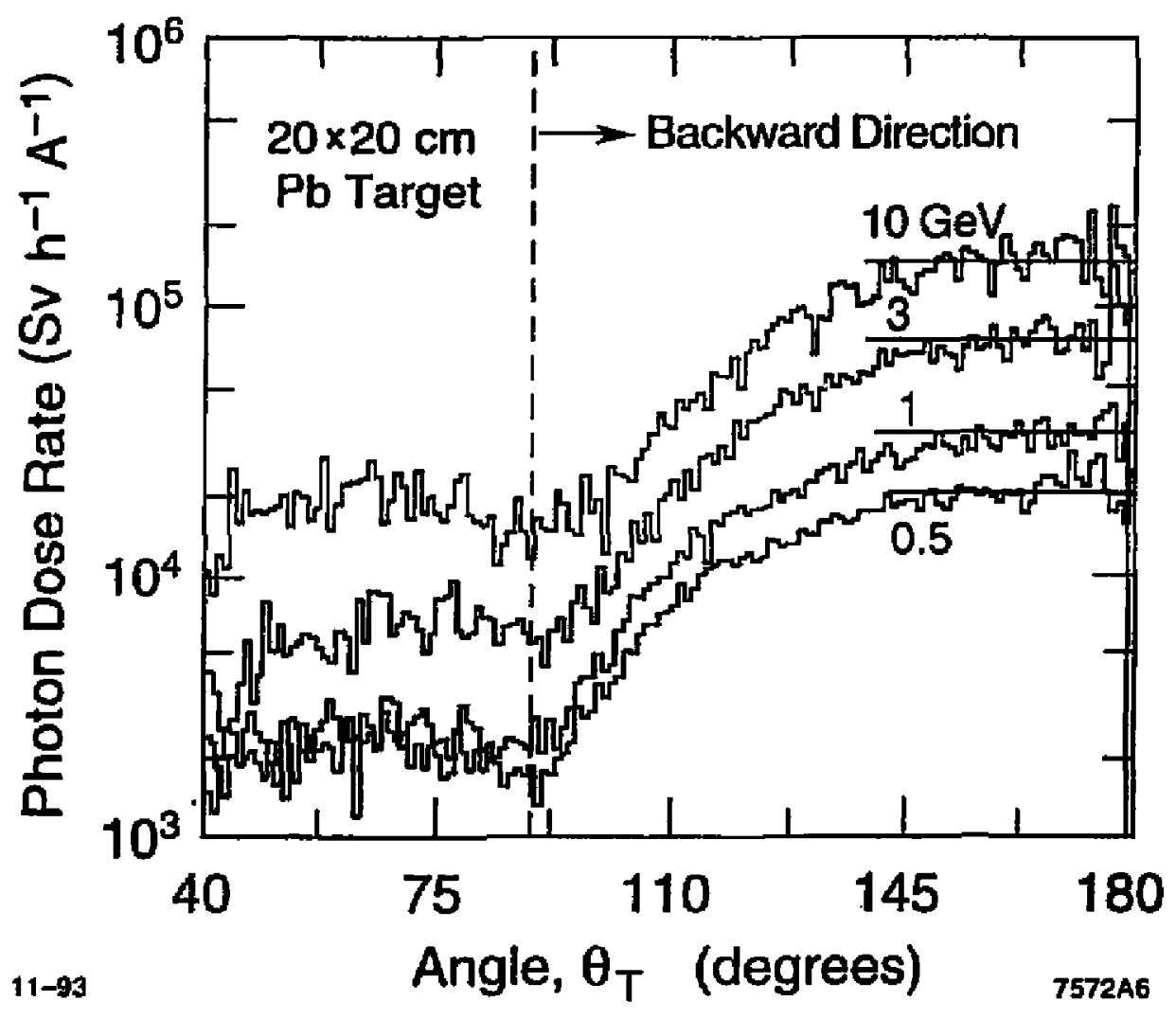

Fig. 6 


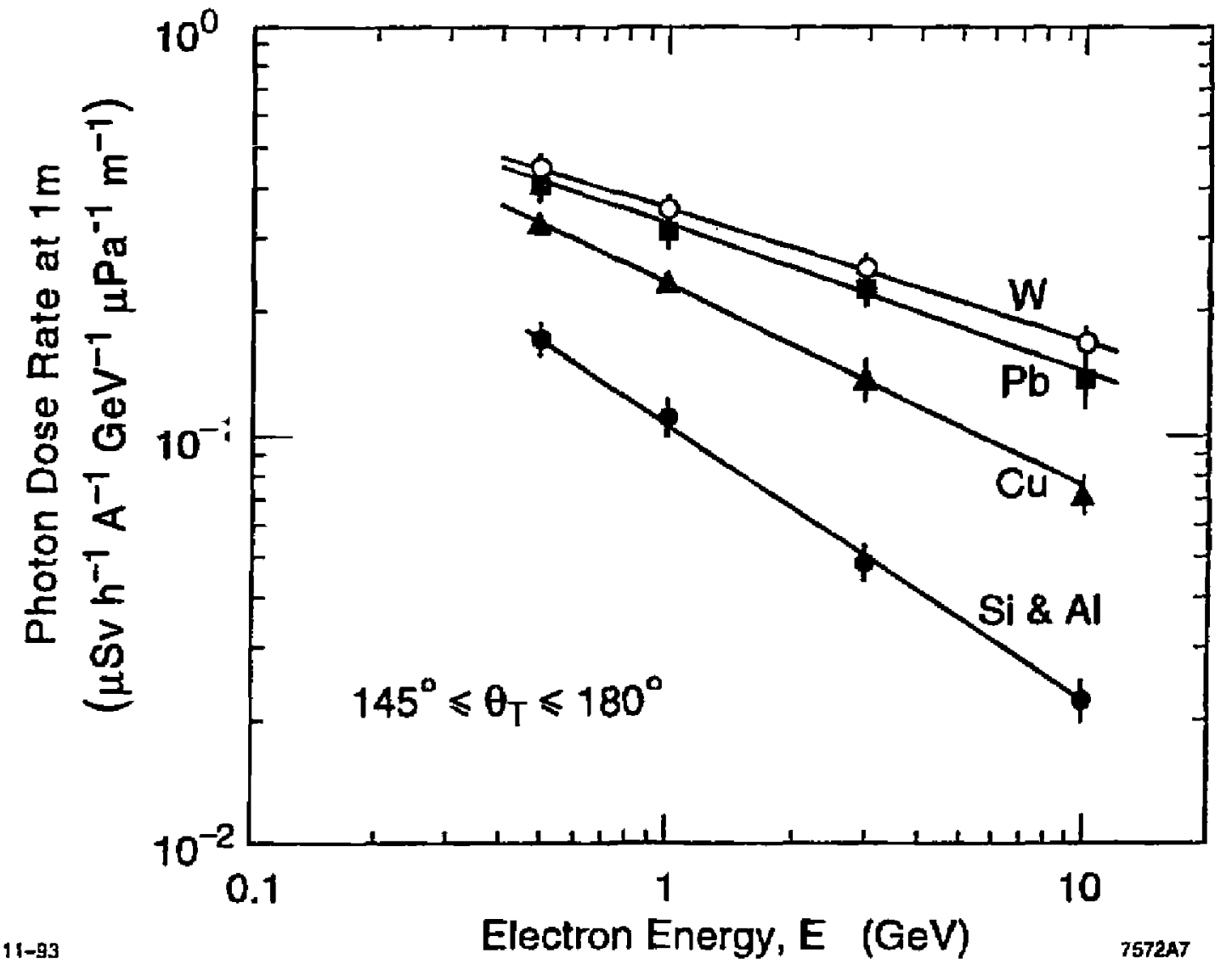

Fig. 7 


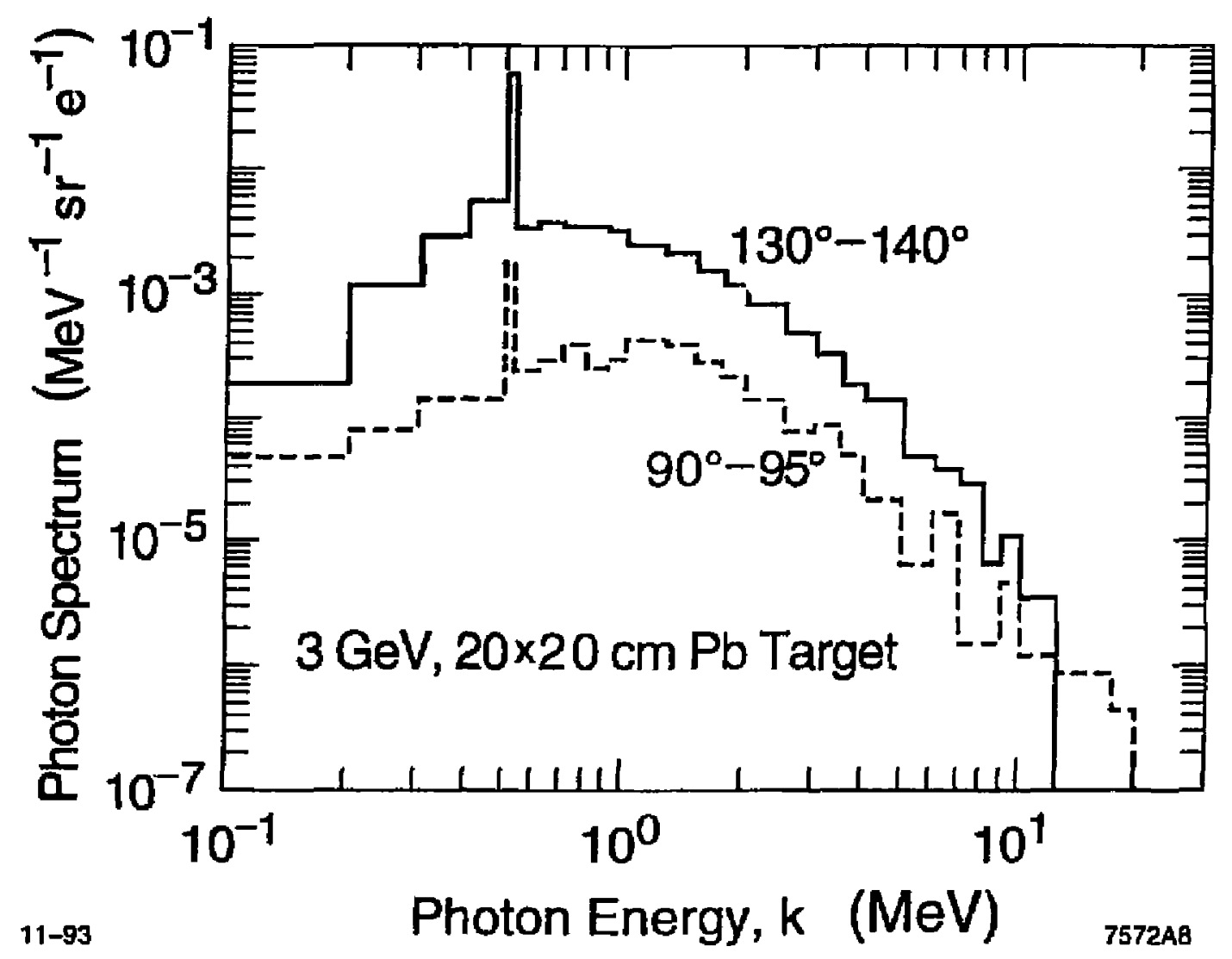

Fig. 8 


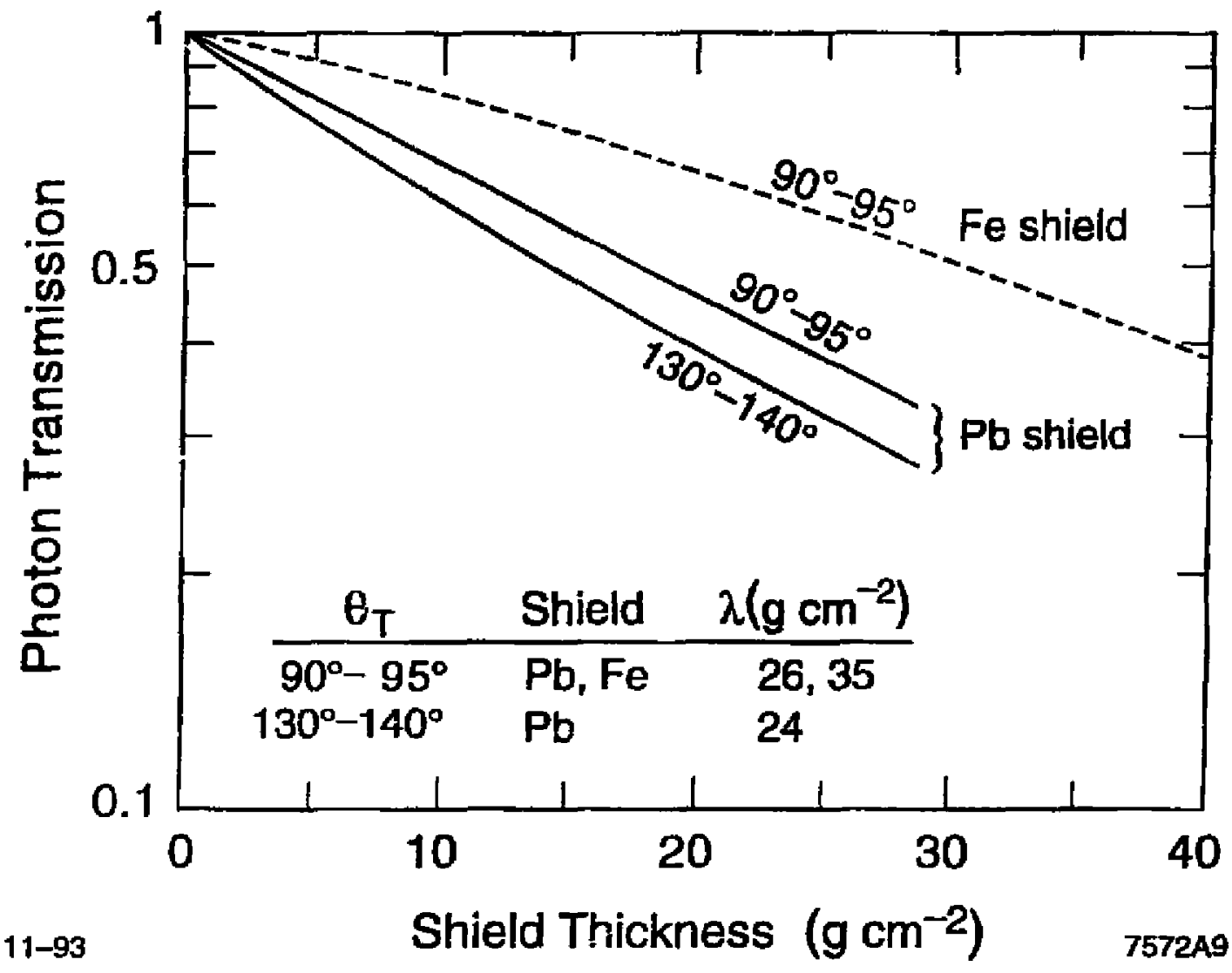

Fig. 9 


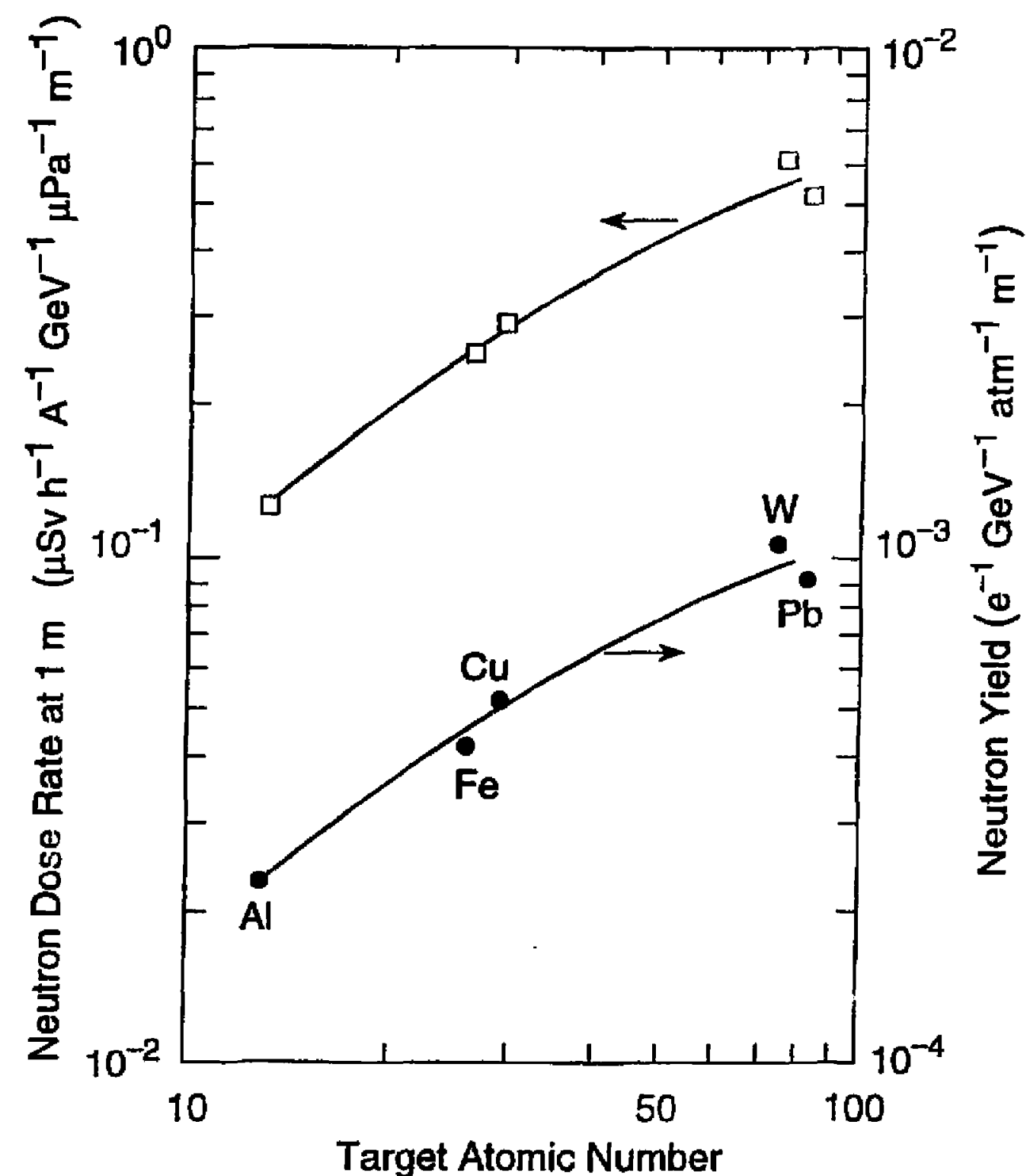

11-93

7572A10

Fig. 10 


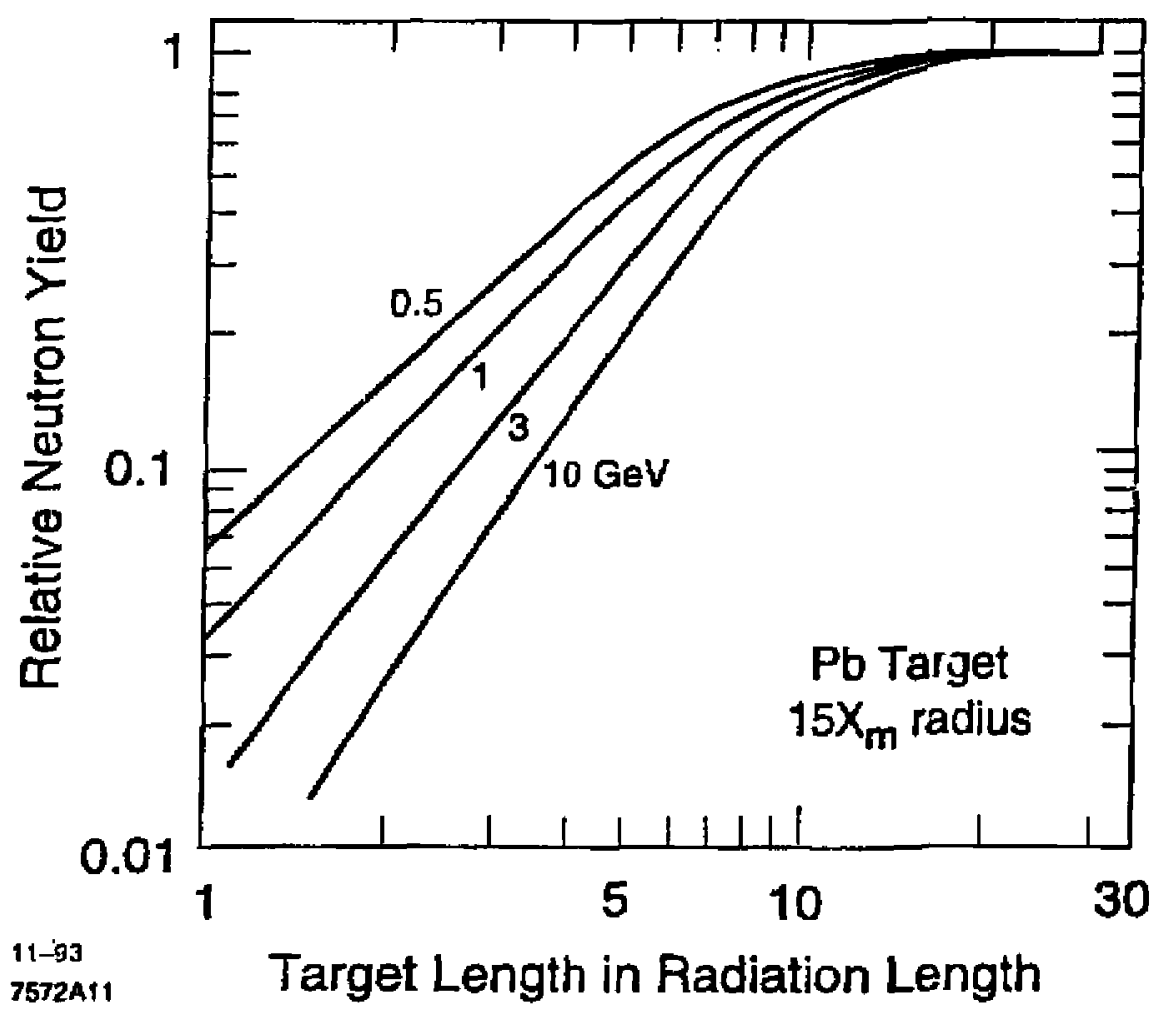

Fig. II 


\begin{tabular}{|c|c|c|c|c|c|c|}
\hline \multirow[b]{2}{*}{ Device } & \multicolumn{3}{|c|}{ Photon } & \multicolumn{3}{|c|}{ Neutron } \\
\hline & Measured & EGS & $\overline{\operatorname{Eqn}(3)}$ & Measured & EGS4 & $\begin{array}{l}\text { Scaling } \\
\text { Method }\end{array}$ \\
\hline $\begin{array}{c}\text { Copper } \\
\text { Slits }\end{array}$ & 0.2 & 0.3 & 0.1 & 0.2 & 0.2 & 0.1 \\
\hline $\begin{array}{l}\text { Tungsten } \\
\text { Beamstop }\end{array}$ & 0.2 & 0.1 & 0.2 & 0.3 & 0.5 & 0.4 \\
\hline
\end{tabular}

1). Errors of the measurements are about a factor of two (dominated by the uncertainty of vacuum pressure.

2). Error of the calculations are about $50 \%$ (dominated by the error in the modeling of the true geometry). 